\title{
Article \\ Direct Transport to Cardiac Arrest Center and Survival Outcomes after Out-of-Hospital Cardiac Arrest by Urbanization Level
}

\author{
Eujene Jung ${ }^{1,2}$, Young Sun Ro ${ }^{3,4,5, * \mathbb{D}}$, Jeong Ho Park ${ }^{3,4,5} \mathbb{D}$, Hyun Ho Ryu ${ }^{1,2,5}$ and Sang Do Shin ${ }^{3,4,5}$ \\ 1 Department of Emergency Medicine, Chonnam National University Hospital, Gwangju 61469, Korea; \\ 81823ej@hanmail.net (E.J.); oriryu@hanmail.net (H.H.R.) \\ 2 Department of Emergency Medicine, Chonnam National University Medical School, Gwangju 61469, Korea \\ 3 Department of Emergency Medicine, Seoul National University Hospital, Seoul 03080, Korea; \\ timthe@gmail.com (J.H.P.); sdshin@snu.ac.kr (S.D.S.) \\ 4 Department of Emergency Medicine, Seoul National University College of Medicine, Seoul 03080, Korea \\ 5 Laboratory of Emergency Medical Services, Seoul National University Hospital Biomedical Research Institute, \\ Seoul 03080, Korea \\ * Correspondence: ro.youngsun@gmail.com; Tel.: +82-2-2072-3257
}

Citation: Jung, E.; Ro, Y.S.; Park, J.H.; Ryu, H.H.; Shin, S.D. Direct Transport to Cardiac Arrest Center and Survival Outcomes after Out-of-Hospital Cardiac Arrest by Urbanization Level. J. Clin. Med. 2022, 11, 1033. https:// doi.org/10.3390/jcm11041033

Academic Editors: Timur Sellmann and Stephan Marsch

Received: 19 January 2022

Accepted: 11 February 2022

Published: 16 February 2022

Publisher's Note: MDPI stays neutral with regard to jurisdictional claims in published maps and institutional affiliations.

Copyright: (C) 2022 by the authors. Licensee MDPI, Basel, Switzerland. This article is an open access article distributed under the terms and conditions of the Creative Commons Attribution (CC BY) license (https:// creativecommons.org/licenses/by/ $4.0 /)$.

\begin{abstract}
Current guidelines for post-resuscitation care recommend regionalized care at a cardiac arrest center (CAC). Our objectives were to evaluate the effect of direct transport to a CAC on survival outcomes of out-of-hospital cardiac arrests (OHCAs), and to assess interaction effects between CAC and urbanization levels. Adult EMS-treated OHCAs with presumed cardiac etiology between 2015 and 2019 were enrolled. The main exposure was the hospital where OHCA patients were transported by EMS (CAC or non-CAC). The outcomes were good neurological recovery and survival to discharge. Multivariable logistic regression analyses were conducted. Interaction analysis between the urbanization level of the location of arrest (metropolitan or urban/rural area) and the exposure variable was performed. Among the 95,931 study population, 23,292 (24.3\%) OHCA patients were transported directly to CACs. Patients in the CAC group had significantly higher likelihood of good neurological recovery and survival to discharge than the non-CAC group (both $p<0.01$, aORs (95\% CIs): 1.75 (1.63-1.89) and 1.70 (1.60-1.80), respectively). There were interaction effects between $\mathrm{CAC}$ and the urbanization level for good neurological recovery and survival to discharge. Direct transport to CAC was associated with significantly better clinical outcomes compared to non-CAC, and the findings were strengthened in OHCAs occurring in nonmetropolitan areas.
\end{abstract}

Keywords: out-of-hospital cardiac arrest; post-resuscitation care; outcomes

\section{Introduction}

Out-of-hospital cardiac arrest (OHCA) is a major global health problem, with high incidence and poor survival outcomes [1,2]. Despite extensive efforts to increase resuscitation and post-resuscitation care, mortality and disability rates remain high, with only $7-10 \%$ of OHCA patients surviving to discharge and only less than $5 \%$ of those patients discharging with favorable neurological recovery [2,3]. The emergency medical services (EMS) personnel are responsible for on-scene and during-transport resuscitation and transporting of OHCA patients to the appropriate hospital for post-resuscitation care [4].

Regional systems of care involving centralization of post-resuscitation care have been proposed to improve survival outcomes of OHCA, as OHCA is considered to be best treated in regional hospitals with highly resource-intensive treatments such as extracorporeal membrane oxygenation, percutaneous cardiac intervention (PCI), and targeted temperature management (TTM) [5,6]. Current guidelines for post-resuscitation care recommend regionalization to designated cardiac arrest centers (CAC) that can provide $24 \mathrm{~h}$ immediate PCI and can provide TTM [5,7]. However, in recent systematic review and meta-analysis 
studies, it was suggested that while transporting patients directly to a CAC did associate with improved clinical outcomes at hospital discharge, it did not improve 1-month survival outcomes [8,9]. Sudden cardiac arrest is one of the most time-sensitive diseases, and the increased transport time interval in bypassing the nearest hospital to reach the destination hospital may be detrimental for some OHCA patients with specific conditions [10].

It is hypothesized that the direct transport to a CAC would improve overall survival outcomes in OHCA patients, while that effect will vary depending on the urbanization level. The urbanization level affects the distribution of CAC as a surrogate indicator of community, EMS, and resources of hospital resuscitation, and is one of the potential risk factors for survival outcomes of OHCA [11]. The objectives of this study were to evaluate effects of the direct transport to a CAC on clinical outcomes of OHCA patients with presumed cardiac etiology, and to assess whether the effects vary across the urbanization level of the location where OHCA occurred.

\section{Materials and Methods}

\subsection{Study Design and Setting}

This is a cross-sectional study, using a nationwide, population-based prospective registry of OHCAs including all patients transported by EMS in Korea.

Korea has approximately 50 million people living in $100,210 \mathrm{~km}^{2}$, and there are 17 provinces. These areas are subdivided into 229 counties for administrative purposes, including 69 counties in metropolitan cities (median population density: 9214 persons per $\mathrm{km}^{2}$ ), 78 counties in urban cities (median population density: 598 persons per $\mathrm{km}^{2}$ ), and 82 counties in rural areas (median population density: 65 persons per $\mathrm{km}^{2}$ ) [12]. Of the 17 provinces, 7 provinces are metropolitan cities, and 10 provinces have a mix of urban cities and rural areas.

The EMS system of Korea is a government-based system operated by 17 provincial headquarters of the National Fire Agency. The EMS personnel perform basic life support on scene and during transport with advanced airway management and intravenous fluid administration. Since declaration of death in the field is not permitted for EMS providers, all EMS-treated OHCA victims are transported to the nearest emergency department (ED) based on the standard operation protocol.

In Korea, there are 402 EDs that are categorized into three levels by the government according to capacity and resources such as equipment, staffing, and size of the ED: level-1 EDs $(n=38)$, level-2 EDs $(n=128)$, and level-3 EDs $(n=236)$. All EDs generally perform acute cardiac management and post-resuscitation care in accordance with international standard guidelines such as the 2020 American Heart Association guidelines [13]. Most of the level-1 and level-2 EDs perform post-resuscitation care such as PCI and TTM. The Ministry of Health and Welfare has designated and operated cardiovascular disease regional centers but has not yet designated and/or certified cardiac arrest-specific regional centers.

\subsection{Data Sources}

This study identified a study population using the Korean nationwide OHCA registry, which captures all EMS-assessed OHCA patients across the country. The OHCA registry is a prospective observational registry that was launched in 2006 through a collaboration between the National Fire Agency and the Korean centers for Disease Control and Prevention (CDC). The registry includes ambulance run sheets, dispatch records, EMS cardiac arrest in-depth registry, and medical record reviews. The medical record reviewers from Korea CDC extract data regarding the etiology, hospital care, and outcomes based on the Utstein guidelines. The project quality management committee (QMC) is composed of emergency medicine physicians, cardiologists, epidemiologists, statistical experts, and medical record review experts. All of the items, including definitions, inclusion and exclusion criteria, examples, and warnings, are defined in the medical record review guidelines and were developed by the project QMC. Explanations on nationwide OHCA registry, detailed data collection process, and quality management protocols are reported in previous studies [14]. 


\subsection{Study Population}

This study included all EMS-treated OHCA patients aged 18 or over with presumed cardiac etiology between January 2015 and December 2019. Patients whose arrest occurred in an ambulance during transport or witnessed by EMS personnel were excluded. The cause of arrest was presumed to be cardiac if there was no evident noncardiac cause such as asphyxia, drowning, trauma, poisoning, and burn. The cause of arrest was measured by medical record reviewers with discharge summary abstracts or medical records written by the inpatient care doctors, based on the Utstein guidelines [15].

\subsection{Main Outcomes}

The primary and secondary outcomes were good neurological recovery and survival to discharge. Good neurological recovery was defined as cerebral performance category I (good cerebral performance; no neurologic disability) or II (moderate cerebral disability; able to perform daily activities independently) at time of hospital discharge.

\subsection{Measurements and Variables}

The main exposure of this study is the type of hospital that patients are transported to by EMS providers, which is classified as either CAC or non-CAC. In this study, CAC was defined as a hospital that performed both PCI and TTM at least once each year during the study period for OHCA patients [5].

Patient arrest information, including age, sex, comorbidities (diabetes mellitus, hypertension, and heart disease), urbanization level of arrest location (metropolitan or urban/rural area), and place of arrest (public or private), were collected. Prehospital EMS information including witness status, bystander CPR, initial electrocardiogram rhythm (shockable or non-shockable), EMS time variables (response time interval (time from the call to ambulance arrival at the scene), scene time interval (time from ambulance arrival to departure from the scene), and transport time interval (time from departure to hospital arrival)), multitier response, prehospital airway management, and mechanical CPR were retrieved. Information concerning hospital outcome-related variables, including post-resuscitation care and clinical outcomes, were also collected.

\subsection{Statistical Analysis}

A descriptive analysis was conducted to compare the characteristics of patients transported to CAC and non-CAC. Categorical variables were shown by counts and proportion and tested by chi-square test. Continuous variables were shown by medians and quartiles and tested by Wilcoxon rank-sum test, since EMS time variables have a nonparametric distribution.

Both univariable and multivariable logistic regression analysis were performed to estimate the effect of direct transport to CACs on study outcomes. Crude and adjusted odds ratios (aORs) with 95\% confidence intervals (CIs) were calculated. Finally, the interaction model between the transported hospital and urbanization level of arrest location (metropolitan area or urban/rural area) was conducted to estimate whether the effects of direct transport to CACs varies across urbanization levels that affect the distribution of CAC.

A sensitivity analysis was conducted for pulseless OHCA patients who did not achieve prehospital return of spontaneous circulation (ROSC), to examine whether the association between direct transport to CAGs and study outcomes were maintained.

All statistical analysis was performed using SAS version 9.4 (SAS Institute Inc., Cary, NC, USA). $p<0.05$ was considered statistically significant.

\subsection{Ethics Statements}

This study complies with the Declaration of Helsinki. This study was approved by the Institutional Review Board (IRB) of Seoul National University Hospital and the requirement 
for informed consent was waived due to the retrospective nature of this study (IRB No. SNUH-1103-153-357).

\section{Results}

Among 139,212 EMS-treated OHCA cases that occurred within the study period, 95,931 (68.9\%) met the inclusion criteria. We excluded patients who were younger than 18 years old $(n=3124)$, who had noncardiac etiology $(n=33,004)$, and those whose arrest occurred during transport $(n=7153)$ (Figure 1$)$.

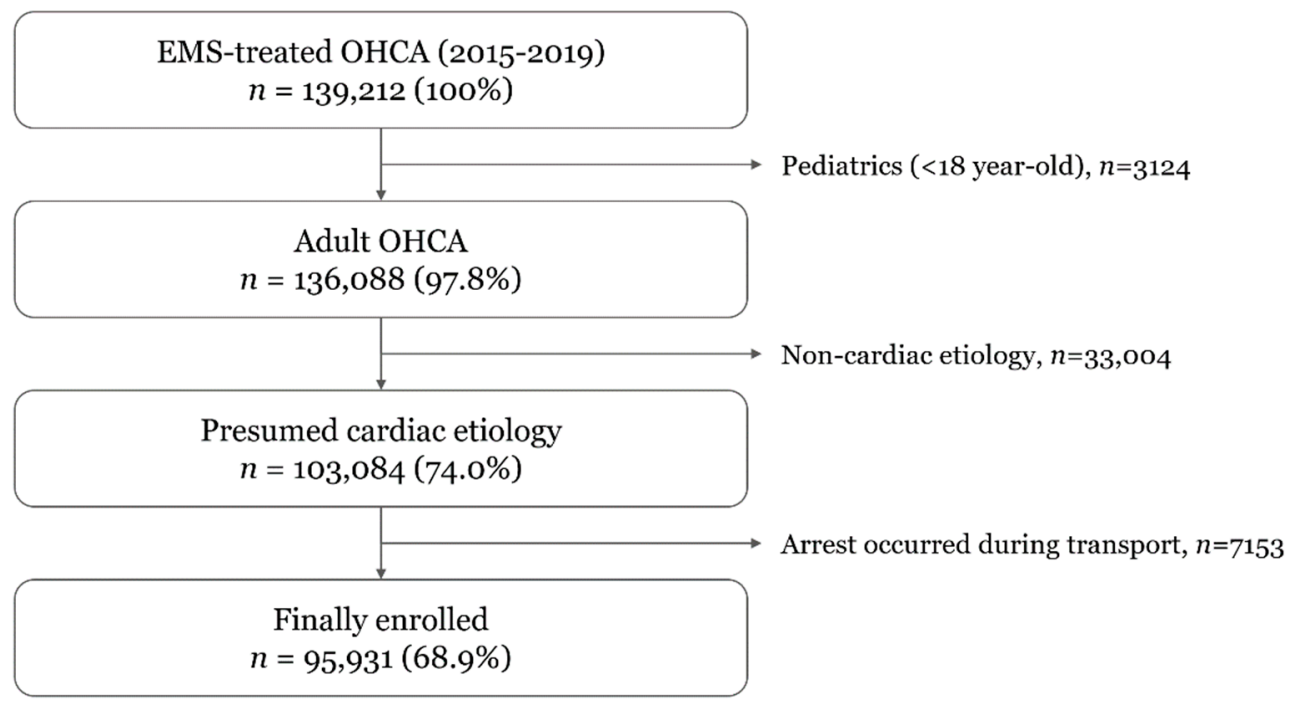

Figure 1. Patient flow. EMS, emergency medical services; OHCA, out-of-hospital cardiac arrest.

\subsection{Demographic Findings}

The demographics of the study population according to the transported hospitals are presented in Table 1. Of the 95,931 eligible patients, $23,292(24.3 \%)$ and $72,639(75.7 \%)$ OHCA patients were transported to CAC and non-CAC hospitals, respectively. The good neurological recovery and survival to discharge rates were $9.1 \%$ and $13.4 \%$ in the CAC group, and $4.7 \%$ and $7.4 \%$ in the non-CAC group, respectively (both $p$-value $<0.01$ ). OHCAs in the CAC group occurred more frequently in metropolitan areas compared to the nonCAC group $(67.1 \%$ vs. $32.1 \%, p<0.01)$, and hospital treatments including TTM, PCI, and ECMO were also performed more frequently in the CAC group. The medians (interquartile ranges) of EMS transport time interval from scene to hospital were 7 (5-10) $\mathrm{min}$ in the CAC group and $6(4-11) \mathrm{min}$ in the non-CAC group $(p<0.01)$.

The demographics of OHCA patients according to the urbanization level of arrest location are summarized in Table 2. Of the eligible patients, 38,939 (40.6\%) cases of OHCA occurred in metropolitan areas and 56,992 (59.4\%) cases occurred in nonmetropolitan areas. OHCA cases occurring in metropolitan areas were transported more frequently to a CAC $(40.1 \%$ vs. $13.5 \%, p<0.01)$. Good neurological recovery and survival to discharge rates were $7.0 \%$ and $10.7 \%$ in the metropolitan group, and $5.0 \%$ and $7.6 \%$ in the urban/rural group, respectively (both $p$-value $<0.01$ ).

\subsection{Main Results}

The results of multivariable logistic regression analyses are shown in Table 3. After adjustments for potential confounders, patients who were transported to CAC hospitals had significantly higher likelihood of good neurological recovery at hospital discharge and survival to discharge than those transported to non-CAC hospitals (aORs (95\% CIs): 1.75 (1.63-1.89) and $1.70(1.60-1.80)$, respectively). 
Table 1. Characteristics of out-of-hospital cardiac arrest patients according to transported hospitals.

\begin{tabular}{|c|c|c|c|c|}
\hline & Total & CAC & Non-CAC & $p$-Value \\
\hline & $n(\%)$ & $n(\%)$ & $n(\%)$ & \\
\hline Total & $95,931(100.0)$ & $23,292(100.0)$ & $72,639(100.0)$ & \\
\hline Age, year & & & & $<0.01$ \\
\hline $19-65$ & $31,722(33.1)$ & $8460(36.3)$ & $23,262(32.0)$ & \\
\hline $65-120$ & $64,209(66.9)$ & $14,832(63.7)$ & $49,377(68.0)$ & \\
\hline Sex, female & $35,089(36.6)$ & $8110(34.8)$ & $26,979(37.1)$ & $<0.01$ \\
\hline \multicolumn{5}{|l|}{ Comorbidity } \\
\hline Diabetes mellitus & $21,386(22.3)$ & $5922(25.4)$ & $15,464(21.3)$ & $<0.01$ \\
\hline Hypertension & $32,708(34.1)$ & 8988 (38.6) & $23,720(32.7)$ & $<0.01$ \\
\hline Heart disease & $16,577(17.3)$ & $4523(19.4)$ & $12,054(16.6)$ & $<0.01$ \\
\hline Metropolitan area & $38,939(40.6)$ & $15,619(67.1)$ & $23,320(32.1)$ & $<0.01$ \\
\hline Place of arrest, public & $19,202(20.0)$ & $5070(21.8)$ & $14,132(19.5)$ & $<0.01$ \\
\hline Arrest witnessed & $44,429(46.3)$ & $11,659(50.1)$ & $32,770(45.1)$ & $<0.01$ \\
\hline Bystander CPR & $57,803(60.3)$ & $13,946(59.9)$ & $43,857(60.4)$ & 0.17 \\
\hline Initial shockable rhythm & $16,237(16.9)$ & $4732(20.3)$ & $11,505(15.8)$ & $<0.01$ \\
\hline Response time interval, min & & & & $<0.01$ \\
\hline $0-3$ & $5123(5.3)$ & $1287(5.5)$ & $3836(5.3)$ & \\
\hline $4-7$ & $50,555(52.7)$ & $13,882(59.6)$ & $36,673(50.5)$ & \\
\hline $8-$ & $40,253(42.0)$ & $8123(34.9)$ & $32,130(44.2)$ & \\
\hline Median (IQR) & $7(5-9)$ & $7(5-10)$ & $7(5-10)$ & $<0.01$ \\
\hline Scene time interval, $\min$ & & & & $<0.01$ \\
\hline $0-10$ & $28,566(29.8)$ & $5761(24.7)$ & $22,805(31.4)$ & \\
\hline $11-15$ & $33,584(35.0)$ & $8280(35.5)$ & $25,304(34.8)$ & \\
\hline $16-$ & $33,781(35.2)$ & 9251 (39.7) & $24,530(33.8)$ & \\
\hline Median (IQR) & $13(10-18)$ & $14(11-18)$ & $13(10-17)$ & $<0.01$ \\
\hline Transport time interval, min & & & & $<0.01$ \\
\hline $0-3$ & $17,312(18.0)$ & 3184 (13.7) & $14,128(19.4)$ & \\
\hline $4-7$ & $39,459(41.1)$ & $10,588(45.5)$ & $28,871(39.7)$ & \\
\hline $8-$ & $39,160(40.8)$ & $9520(40.9)$ & $29,640(40.8)$ & \\
\hline Median (IQR) & $6(4-10)$ & $7(5-10)$ & $6(4-11)$ & $<0.01$ \\
\hline Multitier response & $55,631(58.0)$ & $16,304(70.0)$ & $39,327(54.1)$ & $<0.01$ \\
\hline \multicolumn{5}{|l|}{ EMS management } \\
\hline Advanced airway & $59,978(62.5)$ & $16,650(71.5)$ & $43,328(59.6)$ & $<0.01$ \\
\hline Mechanical CPR & $10,866(11.3)$ & $4138(17.8)$ & $6728(9.3)$ & $<0.01$ \\
\hline ED level & & & & $<0.01$ \\
\hline Level 1 & $17,778(18.5)$ & $10,032(43.1)$ & $7746(10.7)$ & \\
\hline Level 2 & $45,389(47.3)$ & $13,116(56.3)$ & $32,273(44.4)$ & \\
\hline Level 3 & $32,764(23.2)$ & $144(0.6)$ & $32,630(44.9)$ & \\
\hline \multicolumn{5}{|l|}{ Hospital treatment } \\
\hline TTM & $2938(3.1)$ & $1874(8.0)$ & $1064(1.5)$ & $<0.01$ \\
\hline PCI & $5879(6.1)$ & $2521(10.8)$ & $3358(4.6)$ & $<0.01$ \\
\hline ECMO & $936(1.0)$ & $476(2.0)$ & $460(0.6)$ & $<0.01$ \\
\hline \multicolumn{5}{|l|}{ Survival outcomes } \\
\hline Survival to discharge & $8465(8.8)$ & $3120(13.4)$ & $5345(7.4)$ & $<0.01$ \\
\hline Good neurological recovery & $5563(5.8)$ & $2113(9.1)$ & $3450(4.7)$ & $<0.01$ \\
\hline
\end{tabular}

$\mathrm{CAC}$, cardiac arrest center; $\mathrm{CPR}$, cardiopulmonary resuscitation; IQR, interquartile range; ED, emergency department; TTM, targeted temperature management; PCI, percutaneous coronary intervention; ECMO, extracorporeal membrane oxygenation.

\subsection{Interaction Analysis}

In the interaction analysis, statistically significant interaction effects were found between transported hospital and urbanization level (Table 4). The aORs for the outcomes of the CAC group and non-CAC differed depending on the urbanization level of area of OHCA. The CAC hospitals had interaction effects for good neurological recovery at discharge (aORs (95\% CIs): 1.51 (1.40-1.63) for patients in metropolitan areas vs. 1.98 (1.81-2.17) for patients in urban/rural areas, and survival to discharge (aORs (95\% CIs): 
$1.63(1.48-1.80)$ for patients in metropolitan areas vs. 1.91 (1.71-2.14) for patients in ur$\mathrm{ban} /$ rural areas (both $\mathrm{p}$ for interaction $<0.01$ ).

Table 2. Characteristics of out-of-hospital cardiac arrest patients according to urbanization level of arrest location.

\begin{tabular}{|c|c|c|c|c|}
\hline & Total & Metropolitan & Urban/Rural & $p$-Value \\
\hline & $n(\%)$ & $n(\%)$ & $n(\%)$ & \\
\hline Total & $95,931(100.0)$ & $38,939(100.0)$ & $56,992(100.0)$ & \\
\hline Cardiac arrest center & & & & $<0.01$ \\
\hline Yes & $23,292(24.3)$ & $15,619(40.1)$ & $7673(13.5)$ & \\
\hline No & $72,639(75.7)$ & $23,320(59.9)$ & $49,319(86.5)$ & \\
\hline Age, year & & & & $<0.01$ \\
\hline $19-65$ & $23,292(24.3)$ & $15,619(40.1)$ & $7673(13.5)$ & \\
\hline $65-120$ & $72,639(75.7)$ & $23,320(59.9)$ & $49,319(86.5)$ & \\
\hline Sex, female & $64,209(66.9)$ & $25,674(65.9)$ & $38,535(67.6)$ & $<0.01$ \\
\hline \multicolumn{5}{|l|}{ Comorbidity } \\
\hline Diabetes mellitus & $35,089(36.6)$ & $13,801(35.4)$ & $21,288(37.4)$ & $<0.01$ \\
\hline Hypertension & $21,386(22.3)$ & $9441(24.2)$ & $11,945(21.0)$ & $<0.01$ \\
\hline Heart disease & $32,708(34.1)$ & $14,135(36.3)$ & $18,573(32.6)$ & $<0.01$ \\
\hline Place of arrest, public & $19,202(20.0)$ & $8008(20.6)$ & $11,194(19.6)$ & $<0.01$ \\
\hline Arrest witnessed & $44,429(46.3)$ & $18,409(47.3)$ & $26,020(45.7)$ & $<0.01$ \\
\hline Bystander CPR & $57,803(60.3)$ & $22,538(57.9)$ & $35,265(61.9)$ & $<0.01$ \\
\hline Initial shockable rhythm & $16,237(16.9)$ & $7086(18.2)$ & $9151(16.1)$ & 0.01 \\
\hline Response time interval, min & & & & $<0.01$ \\
\hline $0-3$ & $5123(5.3)$ & $2269(5.8)$ & $2854(5.0)$ & \\
\hline $4-7$ & $50,555(52.7)$ & $25,074(64.4)$ & $25,481(44.7)$ & \\
\hline $8-$ & $40,253(42.0)$ & $11,596(29.8)$ & $28,657(50.3)$ & \\
\hline Median (IQR) & $7(5-9)$ & $6(5-8)$ & $8(6-11)$ & $<0.01$ \\
\hline Scene time interval, $\min$ & & & & $<0.01$ \\
\hline $0-10$ & $28,566(29.8)$ & $10,677(27.4)$ & $17,889(31.4)$ & \\
\hline $11-15$ & $33,584(35.0)$ & $15,132(38.9)$ & $18,452(32.4)$ & \\
\hline $16-$ & $33,781(35.2)$ & $13,130(33.7)$ & $20,651(36.2)$ & \\
\hline Median (IQR) & $13(10-18)$ & $13(10-17)$ & $13(10-18)$ & $<0.01$ \\
\hline Transport time interval, min & & & & $<0.01$ \\
\hline $0-3$ & $17,312(18.0)$ & $7366(18.9)$ & $9946(17.5)$ & \\
\hline $4-7$ & $39,459(41.1)$ & $19,765(50.8)$ & $19,694(34.6)$ & \\
\hline $8-$ & $39,160(40.8)$ & $11,808(30.3)$ & $27,352(48.0)$ & \\
\hline Median (IQR) & $6(4-10)$ & $6(4-8)$ & $7(4-12)$ & $<0.01$ \\
\hline Multitier response & $55,631(58.0)$ & $26,993(69.3)$ & $28,638(50.2)$ & $<0.01$ \\
\hline \multicolumn{5}{|l|}{ EMS management } \\
\hline Advanced airway & $59,978(62.5)$ & $27,384(70.3)$ & $32,594(57.2)$ & $<0.01$ \\
\hline Mechanical CPR & $10,866(11.3)$ & $6947(17.8)$ & $3919(6.9)$ & $<0.01$ \\
\hline ED level & & & & $<0.01$ \\
\hline Level 1 & $17,778(18.5)$ & $7336(18.8)$ & $10,442(18.3)$ & \\
\hline Level 2 & $45,389(47.3)$ & $22,232(57.1)$ & $23,157(40.6)$ & \\
\hline Level 3 & $32,764(23.2)$ & $9371(24.1)$ & $23,393(41.1)$ & \\
\hline \multicolumn{5}{|l|}{ Hospital treatment } \\
\hline TTM & $2938(3.1)$ & $1745(4.5)$ & $1193(2.1)$ & $<0.01$ \\
\hline PCI & $5879(6.1)$ & $3185(8.2)$ & $2694(4.7)$ & $<0.01$ \\
\hline ECMO & $936(1.0)$ & $548(1.4)$ & $388(0.7)$ & $<0.01$ \\
\hline \multicolumn{5}{|l|}{ Survival outcomes } \\
\hline Survival to discharge & $8465(8.8)$ & 4155 (10.7) & $4310(7.6)$ & $<0.01$ \\
\hline Good neurological recovery & $5563(5.8)$ & $2711(7.0)$ & $2852(5.0)$ & $<0.01$ \\
\hline
\end{tabular}


Table 3. Multivariable logistic regression models for study outcomes.

\begin{tabular}{|c|c|c|c|c|c|c|}
\hline & \multirow{2}{*}{$\begin{array}{c}\text { Total } \\
n\end{array}$} & \multicolumn{2}{|c|}{ Outcome } & \multirow{2}{*}{$\begin{array}{c}\text { Model } 1 \\
\text { aOR }(95 \% \text { CI) }\end{array}$} & \multirow{2}{*}{$\begin{array}{c}\text { Model } 2 \\
\text { aOR }(95 \% \text { CI })\end{array}$} & \multirow{2}{*}{$\begin{array}{c}\text { Model } 3 \\
\text { aOR }(95 \% \text { CI) }\end{array}$} \\
\hline & & $n$ & $\%$ & & & \\
\hline \multicolumn{7}{|c|}{ Good neurological recovery } \\
\hline \multicolumn{7}{|c|}{ Transported hospital } \\
\hline Noncardiac center & 72,639 & 3450 & 4.7 & 1.00 & 1.00 & 1.00 \\
\hline Cardiac center & 23,292 & 2113 & 9.1 & $1.81(1.70-1.90)$ & $1.66(1.55-1.79)$ & $1.75(1.63-1.89)$ \\
\hline \multicolumn{7}{|l|}{ Urbanization level } \\
\hline Urban/rural area & 56,992 & 2852 & 5.0 & 1.00 & 1.00 & 1.00 \\
\hline Metropolitan area & 38,939 & 2711 & 7.0 & $1.18(1.12-1.26)$ & $1.17(1.09-1.25)$ & $1.05(0.98-1.13)$ \\
\hline \multicolumn{7}{|l|}{ Survival to discharge } \\
\hline \multicolumn{7}{|l|}{ Transported hospital } \\
\hline Noncardiac center & 72,639 & 5345 & 7,4 & 1.00 & 1.00 & 1.00 \\
\hline Cardiac center & 23,292 & 3120 & 13.4 & 1.75 (1.67-1.85) & $1.62(1.53-1.72)$ & $1.70(1.60-1.80)$ \\
\hline \multicolumn{7}{|l|}{ Urbanization level } \\
\hline Urban/rural area & 56,992 & 4310 & 7.6 & 1.00 & 1.00 & 1.00 \\
\hline Metropolitan area & 38,939 & 4155 & 10.7 & $1.23(1.18-1.30)$ & $1.23(1.16-1.30)$ & $1.12(1.05-1.18)$ \\
\hline
\end{tabular}

aOR, adjusted odds ratio; CI, confidence interval. Model 1: adjusted for age and sex. Model 2: adjusted for variables in Model 1, comorbidities (diabetes mellitus, hypertension, and heart disease), place of arrest, witness status, bystander CPR, and initial shockable rhythm. Model 3: adjusted for variables in Model 2, response time interval, scene time interval, transport time interval, multitier response, EMS airway management, and mechanical CPR.

Table 4. Interaction analysis between direct transport to cardiac arrest centers and urbanization level.

\begin{tabular}{|c|c|c|c|c|c|}
\hline & \multicolumn{4}{|c|}{ Transported Hospital } & \multirow{3}{*}{$\begin{array}{c}p \text {-for- } \\
\text { Interaction }\end{array}$} \\
\hline & \multirow{2}{*}{ Non-CAC } & \multicolumn{3}{|c|}{ Cardiac Arrest Center } & \\
\hline & & aOR & & & \\
\hline & \multicolumn{5}{|c|}{ Good neurological recovery } \\
\hline Urbanization level & & & & & $<0.01$ \\
\hline Metropolitan area & ref. & 1.51 & 1.40 & 1.63 & \\
\hline Urban/rural area & ref. & 1.98 & 1.81 & 2.17 & \\
\hline \multicolumn{6}{|l|}{ Survival to discharge } \\
\hline Urbanization level & & & & & $<0.01$ \\
\hline Metropolitan area & ref. & 1.63 & 1.48 & 1.80 & \\
\hline Urban/rural area & ref. & 1.91 & 1.71 & 2.14 & \\
\hline
\end{tabular}

$\mathrm{CAC}$, cardiac arrest center; aOR, adjusted odds ratio; $\mathrm{CI}$, confidence interval.

\subsection{Sensitivity Analysis}

The multivariable logistic regression analysis and interaction analysis were performed for pulseless OHCA patients who did not achieve prehospital ROSC (Tables 5 and S1). Pulseless OHCA patients who were transported to CAC hospitals had significantly higher likelihoods of good neurological recovery and survival to discharge (aORs (95\% CIs): 1.49 (1.18-1.87) and 1.45 (1.30-1.61), respectively). In the interaction analysis, the CAC hospitals had interaction effects for good neurological recovery at discharge (aORs $(95 \%$ CIs): 1.36 (1.04-1.77) for patients in metropolitan areas vs. 1.84 (1.22-2.79) for patients in urban/rural areas, and survival to discharge (aORs (95\% CIs): 1.24 (1.09-1.42) for patients in metropolitan areas vs. $1.91(1.60-2.27)$ for patients in urban/rural areas (both $p$ for interaction $<0.01$ ). 
Table 5. Sensitivity analysis of pulseless OHCA patients who did not achieve prehospital ROSC.

\begin{tabular}{|c|c|c|c|c|c|}
\hline & \multicolumn{4}{|c|}{ Transported Hospital } & \multirow{3}{*}{$\begin{array}{c}p \text {-for- } \\
\text { Interaction }\end{array}$} \\
\hline & \multirow{2}{*}{ Non-CAC } & \multicolumn{3}{|c|}{ Cardiac Arrest Center } & \\
\hline & & aOR & & & \\
\hline \multicolumn{6}{|c|}{ Good neurological recovery } \\
\hline Urbanization level & & & & & $<0.01$ \\
\hline Metropolitan area & ref. & 1.36 & 1.04 & 1.77 & \\
\hline Urban/rural area & ref. & 1.84 & 1.22 & 2.79 & \\
\hline \multicolumn{6}{|l|}{ Survival to discharge } \\
\hline Urbanization level & & & & & $<0.01$ \\
\hline Metropolitan area & ref. & 1.24 & 1.09 & 1.42 & \\
\hline Urban/rural area & ref. & 1.91 & 1.60 & 2.27 & \\
\hline
\end{tabular}

\section{Discussion}

Using the Korean national OHCA database, this study discovered that adult OHCA patients with presumed cardiac etiology who were transported to CAC hospitals were more likely to have better survival outcomes compared to patients transported to non-CAC hospitals. In the interaction analysis, OHCAs occurring in urban/rural areas have better clinical outcomes from direct transport to a CAC hospital. These trends were maintained in the sensitivity analysis of pulseless OHCA patients who did not achieve prehospital ROSC. This research contributes to understanding the relationship between regionalization of post-resuscitation care and overall survival outcomes and will help develop strategies to improve survival outcomes in OHCAs that occur in urban/rural areas.

Regionalized systems of post-resuscitation care have been proposed to improve survival outcomes of OHCAs through centralization of highly resource-intensive treatments such as TTM, acute cardiac care including PCI, and multimodal neuro-intensive care. In recent systematic review and meta-analysis, direct transport of OHCA patients to CACs by EMS providers was associated with increased survival outcomes, despite very low certainty of evidence $[8,9]$. In meta-analysis studies, the definition of CAC varied from study to study, and the capability of PCI was essential, while availability for TTM was treated as important. Although the definition of CAC is used in various ways in different countries based on international guidelines [16], direct transport to a PCI-capable hospital increased overall survival and neurological outcomes of OHCAs $[17,18]$. Only $11 \%$ of OHCA patients who were transported to CAC hospitals had received PCI in this study. Even so, the good clinical outcomes of patients in the CAC group had probably been impacted by capability of $\mathrm{PCI}$ as well as the accumulated experience and ability of these CAC hospitals to treat OHCA patients [9].

However, direct transport of OHCA patients to CAC hospitals results in increased transport time interval for some patients [19]. A previous study related to prehospital transport time of OHCAs reported that delaying hospital arrival time by about $14 \mathrm{~min}$ counteracted the potential benefit of transporting them to a PCI-capable center $[20,21]$. Because current studies focusing on the relationship between distance from scene to hospital and clinical outcomes are mainly conducted in urban environments and limited to relatively short transport time intervals, there is insufficient evidence to agree to current guidelines stating to bypass the nearest hospital and transport to CAC hospitals in rural areas where transport distances may be substantially longer [22,23].

In the interaction analysis of the study, OHCAs occurring in nonmetropolitan areas had higher odds of survival outcomes with direct transport to a CAC hospital compared to OHCAs in metropolitan areas ( $p$-for-interaction $<0.01$ ). One hypothesis that may explain this result is that the clinical capabilities of non-CAC hospitals located in nonmetropolitan areas, including manpower, equipment, and facilities, are below those in metropolitan area. As the beneficial effects of regionalization of post-resuscitation care for OHCAs have been strengthened in urban/rural areas, CACs should be designated and invested in to achieve 
centralization of resource-intensive care to improve the survival outcomes of OHCAs in nonmetropolitan areas [24].

Characteristics of destination hospitals are associated with the clinical outcomes of OHCA, including level of EDs, urbanization level of location of hospitals, teaching status, and OHCA case volume [25-28]. In this study, direct transport of OHCA patients to a hospital where post-resuscitation care is capable showed favorable survival and neurological outcomes, and these results were reinforced in OHCA patients occurring in nonmetropolitan (urban/rural) areas. To improve the survival outcomes of OHCAs, it is considerable to designate and operate CACs to achieve a centralization of resourceintensive post-resuscitation care in nonmetropolitan areas.

This study has a number of limitations. First, since there were no designated cardiac arrest-specific regional centers in Korea, CAC was defined as a hospital that performed PCI and TTM in this study. The definition of CAC varied from study to study [8], which meant the definition would have affected the study results. The generalizability of findings of this study to other countries needs to be further evaluated. Second, the definition of urbanization level (metropolitan vs. urban/rural area) may not accurately reflect the level of medical resources because it is population-based and therefore such classification may have influenced the study results. Third, it is difficult to perform a geospatial analysis to evaluate the proportion of OHCA patients who were not transported to the nearest ED and their EMS transport time interval in this study. Fourth, this study is an observational retrospective analysis, which may have introduced some unmeasured confounders as is known with this study design. Lastly, while we used multivariable analysis, unmeasured and unmeasurable confounders may have influenced the clinical outcomes of the study.

\section{Conclusions}

Direct transport of OHCA patients to cardiac arrest centers was associated with significantly higher survival and favorable neurological outcomes compared to patients transported to non-CAC hospitals. Furthermore, the findings were consistent and strengthened in OHCAs occurring in nonmetropolitan areas. Designating and investing in CACs to achieve centralization of post-resuscitation care could improve the survival outcomes of OHCAs, especially in nonmetropolitan areas.

Supplementary Materials: The following supporting information can be downloaded at: https: / / www.mdpi.com/article/10.3390/jcm11041033/s1. Table S1: Sensitivity analysis of multivariable logistic regression model for pulseless out-of-hospital cardiac arrest patients who did not achieve prehospital ROSC.

Author Contributions: Conceptualization: E.J. and Y.S.R.; Data curation: E.J. and J.H.P.; Formal analysis: E.J.; Investigation: J.H.P. and S.D.S.; Methodology: Y.S.R. and S.D.S.; Software: E.J.; Supervision: Y.S.R., H.H.R. and S.D.S.; Validation: J.H.P., H.H.R. and S.D.S.; Visualization: E.J.; Writing-original draft: E.J.; Writing-review and editing: Y.S.R. All authors have read and agreed to the published version of the manuscript.

Funding: This research received no external funding.

Institutional Review Board Statement: This study complies with the Declaration of Helsinki. This study was approved by the Institutional Review Board (IRB) of Seoul National University Hospital and the requirement for informed consent was waived due to the retrospective nature of this study (IRB No. SNUH-1103-153-357).

Informed Consent Statement: Patient consent was waived due to anonymized data.

Data Availability Statement: The data of this study were obtained from the Korea Centers for Disease Control and Prevention, but restrictions apply to the availability of these data and so are not publicly available.

Conflicts of Interest: The authors declare no conflict of interest. 


\section{References}

1. Atwood, C.; Eisenberg, M.S.; Herlitz, J.; Rea, T.D. Incidence of EMS-treated out-of-hospital cardiac arrest in Europe. Resuscitation 2005, 67, 75-80. [CrossRef] [PubMed]

2. Berdowski, J.; Berg, R.A.; Tijssen, J.G.; Koster, R.W. Global incidences of out-of-hospital cardiac arrest and survival rates: Systematic review of 67 prospective studies. Resuscitation 2010, 81, 1479-1487. [CrossRef] [PubMed]

3. Sasson, C.; Rogers, M.A.; Dahl, J.; Kellermann, A.L. Predictors of survival from out-of-hospital cardiac arrest: A systematic review and meta-analysis. Circ. Cardiovasc. Qual. Outcomes 2010, 3, 63-81. [CrossRef] [PubMed]

4. Hansen, C.M.; Kragholm, K.; Granger, C.B.; Pearson, D.A.; Tyson, C.; Monk, L.; Corbett, C.; Nelson, R.D.; Dupre, M.E.; Fosbøl, E.L. The role of bystanders, first responders, and emergency medical service providers in timely defibrillation and related outcomes after out-of-hospital cardiac arrest: Results from a statewide registry. Resuscitation 2015, 96, 303-309. [CrossRef] [PubMed]

5. Nolan, J.P.; Soar, J.; Cariou, A.; Cronberg, T.; Moulaert, V.R.; Deakin, C.D.; Bottiger, B.W.; Friberg, H.; Sunde, K.; Sandroni, C. European resuscitation council and European society of intensive care medicine 2015 guidelines for post-resuscitation care. Intensive Care Med. 2015, 41, 2039-2056. [CrossRef] [PubMed]

6. Berg, K.M.; Cheng, A.; Panchal, A.R.; Topjian, A.A.; Aziz, K.; Bhanji, F.; Bigham, B.L.; Hirsch, K.G.; Hoover, A.V.; Kurz, M.C. Part 7: Systems of Care: 2020 American Heart Association Guidelines for Cardiopulmonary Resuscitation and Emergency Cardiovascular Care. Circulation 2020, 142, S580-S604. [CrossRef]

7. Merchant, R.M.; Topjian, A.A.; Panchal, A.R.; Cheng, A.; Aziz, K.; Berg, K.M.; Lavonas, E.J.; Magid, D.J. Part 1: Executive Summary: 2020 American Heart Association Guidelines for Cardiopulmonary Resuscitation and Emergency Cardiovascular Care. Circulation 2020, 142, S337-S357. [CrossRef]

8. Yeung, J.; Matsuyama, T.; Bray, J.; Reynolds, J.; Skrifvars, M.B. Does care at a cardiac arrest centre improve outcome after out-of-hospital cardiac arrest? - A systematic review. Resuscitation 2019, 137, 102-115. [CrossRef] [PubMed]

9. Lipe, D.; Giwa, A.; Caputo, N.D.; Gupta, N.; Addison, J.; Cournoyer, A. Do Out-of-Hospital Cardiac Arrest Patients Have Increased Chances of Survival When Transported to a Cardiac Resuscitation Center? J. Am. Heart Assoc. 2018, 7, e011079. [CrossRef] [PubMed]

10. Cha, W.C.; Lee, S.C.; Do Shin, S.; Song, K.J.; Sung, A.J.; Hwang, S.S. Regionalisation of out-of-hospital cardiac arrest care for patients without prehospital return of spontaneous circulation. Resuscitation 2012, 83, 1338-1342. [CrossRef]

11. Yasunaga, H.; Miyata, H.; Horiguchi, H.; Tanabe, S.; Akahane, M.; Ogawa, T.; Koike, S.; Imamura, T. Population density, call-response interval, and survival of out-of-hospital cardiac arrest. Int. J. Health Geogr. 2011, 10, 26. [CrossRef] [PubMed]

12. Jung, E.; Ro, Y.S.; Ryu, H.H.; Shin, S.D.; Moon, S. Interaction Effects between COVID-19 Outbreak and Community Income Levels on Excess Mortality among Patients Visiting Emergency Departments. J. Korean Med. Sci. 2021, 36, e100. [CrossRef] [PubMed]

13. Magid, D.J.; Aziz, K.; Cheng, A.; Hazinski, M.F.; Hoover, A.V.; Mahgoub, M.; Panchal, A.R.; Sasson, C.; Topjian, A.A.; Rodriguez, A.J.; et al. Part 2: Evidence Evaluation and Guidelines Development: 2020 American Heart Association Guidelines for Cardiopulmonary Resuscitation and Emergency Cardiovascular Care. Circulation 2020, 142, S358-S365. [CrossRef] [PubMed]

14. Ro, Y.S.; Shin, S.D.; Lee, Y.J.; Lee, S.C.; Song, K.J.; Ryoo, H.W.; Ong, M.E.; McNally, B.; Bobrow, B.; Tanaka, H.; et al. Effect of Dispatcher-Assisted Cardiopulmonary Resuscitation Program and Location of Out-of-Hospital Cardiac Arrest on Survival and Neurologic Outcome. Ann. Emerg. Med. 2017, 69, 52-61. [CrossRef] [PubMed]

15. Perkins, G.D.; Jacobs, I.G.; Nadkarni, V.M.; Berg, R.A.; Bhanji, F.; Biarent, D.; Bossaert, L.L.; Brett, S.J.; Chamberlain, D.; de Caen, A.R.; et al. Cardiac Arrest and Cardiopulmonary Resuscitation Outcome Reports: Update of the Utstein Resuscitation Registry Templates for Out-of-Hospital Cardiac Arrest: A Statement for Healthcare Professionals from a Task Force of the International Liaison Committee on Resuscitation (American Heart Association, European Resuscitation Council, Australian and New Zealand Council on Resuscitation, Heart and Stroke Foundation of Canada, InterAmerican Heart Foundation, Resuscitation Council of Southern Africa, Resuscitation Council of Asia); and the American Heart Association Emergency Cardiovascular Care Committee and the Council on Cardiopulmonary, Critical Care, Perioperative and Resuscitation. Circulation 2014, 132, 1286-1300. [CrossRef]

16. Sinning, C.; Ahrens, I.; Cariou, A.; Beygui, F.; Lamhaut, L.; Halvorsen, S.; Nikolaou, N.; Nolan, J.P.; Price, S.; Monsieurs, K.; et al. The cardiac arrest centre for the treatment of sudden cardiac arrest due to presumed cardiac cause: Aims, function, and structure: Position paper of the ACVC association of the ESC, EAPCI, EHRA, ERC, EUSEM, and ESICM. Eur. Heart J. Acute Cardiovasc. Care 2020, 9, S193-S202. [CrossRef]

17. Stub, D.; Smith, K.; Bray, J.E.; Bernard, S.; Duffy, S.J.; Kaye, D.M. Hospital characteristics are associated with patient outcomes following out-of-hospital cardiac arrest. Heart 2011, 97, 1489-1494. [CrossRef]

18. Wnent, J.; Seewald, S.; Heringlake, M.; Lemke, H.; Brauer, K.; Lefering, R.; Fischer, M.; Jantzen, T.; Bein, B.; Messelken, M.; et al. Choice of hospital after out-of-hospital cardiac arrest-a decision with far-reaching consequences: A study in a large German city. Crit. Care 2012, 16, R164. [CrossRef]

19. Nichol, G.; Aufderheide, T.P.; Eigel, B.; Neumar, R.W.; Lurie, K.G.; Bufalino, V.J.; Callaway, C.W.; Menon, V.; Bass, R.R.; Abella, B.S.; et al. Regional systems of care for out-of-hospital cardiac arrest: A policy statement from the American Heart Association. Circulation 2010, 121, 709-729. [CrossRef]

20. Chien, C.Y.; Tsai, S.L.; Tsai, L.H.; Chen, C.B.; Seak, C.J.; Weng, Y.M.; Lin, C.C.; Ng, C.J.; Chien, W.C.; Huang, C.H. Impact of Transport Time and Cardiac Arrest Centers on the Neurological Outcome After Out-of-Hospital Cardiac Arrest: A Retrospective Cohort Study. J. Am. Heart Assoc. 2020, 9, e015544. [CrossRef] 
21. Cournoyer, A.; Notebaert, É.; de Montigny, L.; Ross, D.; Cossette, S.; Londei-Leduc, L.; Iseppon, M.; Lamarche, Y.; Sokoloff, C.; Potter, B.J. Impact of the direct transfer to percutaneous coronary intervention-capable hospitals on survival to hospital discharge for patients with out-of-hospital cardiac arrest. Resuscitation 2018, 125, 28-33. [CrossRef] [PubMed]

22. Davis, D.P.; Fisher, R.; Aguilar, S.; Metz, M.; Ochs, G.; McCallum-Brown, L.; Ramanujam, P.; Buono, C.; Vilke, G.M.; Chan, T.C.; et al. The feasibility of a regional cardiac arrest receiving system. Resuscitation 2007, 74, 44-51. [CrossRef] [PubMed]

23. Spaite, D.W.; Bobrow, B.J.; Vadeboncoeur, T.F.; Chikani, V.; Clark, L.; Mullins, T.; Sanders, A.B. The impact of prehospital transport interval on survival in out-of-hospital cardiac arrest: Implications for regionalization of post-resuscitation care. Resuscitation $\mathbf{2 0 0 8}$ 79, 61-66. [CrossRef]

24. Girotra, S.; Chan, P.S.; Bradley, S.M. Post-resuscitation care following out-of-hospital and in-hospital cardiac arrest. Heart (Br. Card. Soc.) 2015, 101, 1943-1949. [CrossRef]

25. Carr, B.G.; Goyal, M.; Band, R.A.; Gaieski, D.F.; Abella, B.S.; Merchant, R.M.; Branas, C.C.; Becker, L.B.; Neumar, R.W. A national analysis of the relationship between hospital factors and post-cardiac arrest mortality. Intensive Care Med. 2009, 35, 505-511. [CrossRef] [PubMed]

26. Shin, S.D.; Suh, G.J.; Ahn, K.O.; Song, K.J. Cardiopulmonary resuscitation outcome of out-of-hospital cardiac arrest in low-volume versus high-volume emergency departments: An observational study and propensity score matching analysis. Resuscitation 2011, 82, 32-39. [CrossRef] [PubMed]

27. Søholm, H.; Wachtell, K.; Nielsen, S.L.; Bro-Jeppesen, J.; Pedersen, F.; Wanscher, M.; Boesgaard, S.; Møller, J.E.; Hassager, C.; Kjaergaard, J. Tertiary centres have improved survival compared to other hospitals in the Copenhagen area after out-of-hospital cardiac arrest. Resuscitation 2013, 84, 162-167. [CrossRef]

28. Ro, Y.S.; Shin, S.D.; Song, K.J.; Park, C.B.; Lee, E.J.; Ahn, K.O.; Cho, S.I. A comparison of outcomes of out-of-hospital cardiac arrest with non-cardiac etiology between emergency departments with low- and high-resuscitation case volume. Resuscitation 2012, 83, 855-861. [CrossRef] 